\title{
La co-ingénierie de la participation : une expérience citoyenne sur la rivière Drôme
}

\author{
Émeline Hassenforder ${ }^{1, *} \odot$, Sabine Girard ${ }^{2} \odot$, Nils Ferrand ${ }^{3}$, Claire Petitjean $^{4}$ et Chrystel Fermond ${ }^{5}$ \\ ${ }^{1}$ Sciences de gestion, CIRAD, UMR G-EAU, INRAE, AgroParisTech, IRD, Montpellier SupAgro, Université \\ de Montpellier, Montpellier, France \\ ${ }^{2}$ Géographie, Université de Grenoble Alpes, INRAE, LESSEM, Grenoble, France \\ ${ }^{3}$ Modélisation et aide à la décision, INRAE, UMR G-EAU, AgroParisTech, CIRAD, IRD, Montpellier SupAgro, Université \\ de Montpellier, Montpellier, France \\ ${ }^{4}$ Facilitatrice du projet SPARE, Syndicat mixte de la rivière Drôme et de ses affluents, Saillans, France \\ ${ }^{5}$ Directrice du Syndicat mixte de la rivière Drôme et de ses affluents, Saillans, France
}

Reçu le 6 avril 2018. Accepté le 4 juin 2020

Le présent article relate et analyse une expérience de recherche-intervention qui s'inscrit dans le cadre de la révision du schéma d'aménagement et de gestion des eaux (SAGE) de la Drôme, dont la préparation a commencé en 2016-2017. Les citoyens ont été associés très tôt à l'élaboration du processus participatif et le texte revient sur un ensemble de leviers et de freins à cette participation amont, en adoptant un angle réflexif et critique. Il défend une perspective méthodologique - celle de la co-ingénierie de la participation - basée sur l'outil PrePar, en analyse les effets et en souligne les apports et les limites à partir du cas de la Drôme. C'est cette dimension réflexive, associée à une analyse des interactions entre recherche, société civile et politiques publiques, qui fait toute la richesse et l'originalité de ce texte.

La Rédaction

\begin{abstract}
Résumé - Cet article détaille un processus participatif mis en place sur le bassin versant de la Drôme pour préparer, avec les citoyens, la révision du Schéma d'Aménagement et de Gestion des Eaux. Spécifiquement, des citoyens ont été associés précocement pour choisir et organiser leur propre processus participatif. Cette co-ingénierie les a sensibilisés à l'importance de ces décisions, a initié des apprentissages sociaux et posé les conditions d'une régulation efficiente. Elle a clarifié les liens entre la participation et la gouvernance institutionnelle. Mais l'exercice apparait abstrait aux participants. Il requiert des règles de participation claires et une initiation aux enjeux et au vocabulaire de la participation. En conclusion, nous suggérons des adaptations de la co-ingénierie de la participation en fonction des attentes et moyens disponibles.
\end{abstract}

Mots-clés : environnement / gouvernance / citoyen / planification / apprentissage / procédure

\begin{abstract}
Participatory co-engineering with citizens of the Drôme River Basin. This paper investigates a participatory process set up in the Drôme river basin to get citizens to support the updating of the water management scheme. Some citizens were engaged at an early stage in selecting and structuring their own participatory process. Such co-engineering raised their awareness about the importance of these decisions, fostered social learning and triggered conditions for an efficient regulation. It clarified the links between participation and institutional governance. But co-engineering was abstract for participants. Coengineering requires clear participation rules and sufficient awareness of the issues and vocabulary of participation. To conclude we propose adjustments to the co-engineering of participation according to the expectations and resources available.
\end{abstract}

Keywords: environment / governance / citizen / planning / learning / procedure

*Auteur correspondant : emeline.hassenforder@cirad.fr 
La participation des citoyens à la gestion de l'eau est souvent déclenchée par une organisation, un groupe ou un individu dans un but précis. Cela peut être pour associer des citoyens à la collecte de données, pour faciliter ou récolter des avis sur un projet d'aménagement ou encore pour encourager des changements de pratiques. En général, c'est l'instigateur de la démarche qui s'occupe de l'ingénierie de la participation, c'est-àdire qui définit en amont les objectifs du dispositif participatif et ses étapes, et qui identifie qui va y être associé, quand, et avec quel rôle (Daniell et al., 2010) ${ }^{1}$.

L'ingénierie de la participation, de fait non participative à partir du moment où elle est conduite par l'instigateur de la démarche uniquement, a ainsi le mérite de pouvoir être rapidement opérationnalisée ou déléguée à un consultant. Quelques routines ou méthodes normalisées permettent de tracer les contours du dispositif participatif envisagé. Néanmoins, cette ingénierie non participative est souvent porteuse de lacunes. D'une part, les choix portant sur les catégories de personnes associées, leur implication ou les méthodes participatives, ne sont pas toujours explicités aux participants. Or ces choix sont cruciaux pour la démarche participative et ses résultats. Se mobilisant pour une cause ou un projet, il est légitime que les participants puissent être associés, ou au moins informés de façon transparente, sur l'ingénierie de la démarche qui les engage. D'autre part, cette ingénierie non participative retarde l'intervention citoyenne et limite, de ce fait, la prise en compte potentielle des avis des citoyens dans la politique, le projet ou le programme de gestion de l'eau. Enfin, si cette participation citoyenne vise à accompagner des changements de pratiques, nous supposons que ces changements seront plus effectifs et durables si la démarche suscite l'adhésion des participants. Ce postulat est issu, entre autres, de réflexions en sociologie de la traduction, qui suggèrent également que cette adhésion peut être favorisée par des «centres de traduction» (Callon et Law, 1988, p. 102), c'est-à-dire des «espaces dédiés aux échanges, laissant libre l'expression de la controverse et des points de vue, indispensables pour que les acteurs co-construisent la démarche innovante» (Durand et al., 2018). La création de ces espaces est précisément un des enjeux de l'ingénierie de la participation.

Dans cette perspective, nous avons souhaité tester et évaluer l'impact d'une participation précoce des citoyens à la conception d'un tel processus. Plusieurs auteurs sont

\footnotetext{
${ }^{1}$ Ces constats ne s'appliquent pas forcément à des dispositifs participatifs spontanés, émergents ou contestataires, pour lesquels l'organisation de la participation est plus informelle et réalisée chemin-faisant. Ces derniers ne se situent donc pas directement dans le champ de cet article.
}

favorables à une participation en amont du processus de décision, sans en nier les limites (Barbier, 2005; Floc'Hlay et Plottu, 1998). Dans le cadre du projet européen SPARE ${ }^{2}$, nous avons ainsi initié et accompagné un groupe de citoyens dans une «co-ingénierie de la participation ». La démarche participative s'inscrivait dans le cadre de la révision du schéma d'aménagement et de gestion des eaux (SAGE) de la Drôme et visait à permettre aux citoyens de faire des propositions concrètes à la commission locale de l'eau en amont de cette révision, et en en respectant le déroulement réglementaire. Quarante-six citoyens ont été impliqués dans cette co-ingénierie et ont produit un plan de participation en s'appuyant sur la méthodologie «PrePar» (Hassenforder et al., 2021a).

La question qui nous intéresse dans cet article est de savoir ce qu'apporte aux participants et au processus participatif l'implication des citoyens dès l'étape d'ingénierie de la participation. Nous nous plaçons du point de vue du gestionnaire de l'eau, dans la mesure où ce sont souvent ces derniers qui sont à l'initiative des démarches participatives autour de l'eau en France. Notre analyse porte sur une méthodologie et un contexte spécifiques, ce qui induit nécessairement des limites à la montée en généralité de nos résultats. S'agissant d'une recherche-intervention en contexte politique réel, sur un territoire limité, toute comparaison avec un groupe témoin isolé socialement était impossible. Nous revenons sur ces limites dans la discussion et la conclusion de cet article. Néanmoins, nous arguons que la méthodologie PrePar est suffisamment générique pour que les résultats de cette analyse soient pertinents pour d'autres démarches de co-ingénierie de la participation.

\section{La co-ingénierie de la participation}

\section{Définition}

L'ingénierie de la participation peut être définie comme «une forme de méta-niveau d'ingénierie et de processus décisionnel organisationnel qui définit les règles et les processus de choix collectif dans la politique et la planification de la gestion de l'eau » (Daniell et al., 2010). Concrètement, l'ingénierie se matérialise par une réflexion en vue de décider des objectifs, des participants et de leurs rôles, du choix de méthodes, de la mise en œuvre, du suivi et de l'évaluation du processus participatif. L' « ingénierie participative de la participation»,

\footnotetext{
2 Projet européen Interreg SPARE (Strategic planning for alpine river ecosystems/Planification stratégique pour les écosystèmes des rivières alpines), www.alpine-space.eu/ projects/spare/.
} 
appelée ici co-ingénierie de la participation, se distingue de l'ingénierie participative classique par le fait que cette réflexion est portée par un groupe d'acteurs mixte, incluant aussi de futurs participants. Le groupe peut inclure l'instigateur de la démarche, la facilitatrice, des élus, des spécialistes et tout autre participant ciblé par la démarche participative. Dans cet article, nous parlerons de co-ingénierie lorsque les citoyens sont associés à cette réflexion. Le terme « citoyen » est défini ici comme une personne qui est concernée individuellement par les affaires du territoire (la «cité»). Les citoyens ne représentent aucun enjeu ou intérêt pré-identifié ou localisé, à la différence des parties prenantes telles que les représentants de collectivités, d'associations, d'entreprises, etc. Cette co-ingénierie est donc un processus additionnel au cadre participatif classique. Elle induit un coût complémentaire en matière d'engagement politique, financier, temporel et humain.

\section{Du point de vue opérationnel}

Dans le contexte français où la participation est de plus en plus encouragée et promue par les autorités ${ }^{3}$, un marché des professionnels de la participation se développe (Bherer et al., 2017; Mazeaud, 2012), appuyé par des formations universitaires (masters et formations continues spécialisés sur l'ingénierie de la participation). Les compétences en ingénierie de la participation sont de plus en plus internalisées au sein des administrations et des services de l'État. Ces professionnels développent différentes approches, qu'ils soient des acteurs associa$\mathrm{tifs}^{4}$, des organisations internationales (ex: OECD, 2015; The World Bank, 1996), des chercheurs ${ }^{5}$ ou des consultants (ex: Chemery et al., 2018; Dionnet et al., 2017). Les principes et les étapes de ces différentes approches sont standards: il s'agit d'identifier les

\footnotetext{
$\overline{3}$ Ordonnance $\mathrm{n}^{\mathrm{o}} 2016-1060$ du 3 août 2016 portant réforme des procédures destinées à assurer l'information et la participation du public à l'élaboration de certaines décisions susceptibles d'avoir une incidence sur l'environnement, Journal officiel, 0181, 5 août 2016.

${ }^{4}$ Voir par exemple les fiches produites par le réseau régional d'éducation à l'environnement en Guyane (GRAINE Guyane) (http://graineguyane.org/wp-content/uploads/2017/09/Par tie 2-Preparer-un-projet-participatif-1.pdf), ou la brochure réalisée l'Institut de formation et de recherche en éducation à l'environnement (Ifrée) [https://www.ifree.asso.fr/images/ publications/autres/brochure_dialogue_territorial_lbpageapa ge_web2.pdf].

5 Voir par exemple l'approche CoOPLAGE développée par des chercheurs de l'unité mixte de recherche Gestion de l'eau, acteurs, usages (G-EAU) en France: http://cooplaage.wata game.info/; ou encore $\mathrm{SAS}^{2}$ Dialogue développé par des chercheurs de l'université de Carleton au Canada : Participatory action research, www.participatoryactionresearch.net.
}

objectifs, les acteurs, leurs rôles, les étapes, les méthodes, le calendrier du processus participatif, son lien avec le processus de décision institutionnelle, la communication à mettre en œuvre, etc. C'est également le cas de l'outil méthodologique PrePar (Hassenforder et al., 2021a) que nous avons mobilisé. Les approches d'ingénierie de la participation sont utilisées dans différents domaines, et principalement l'environnement, l'urbanisme et la santé. Cependant, à notre connaissance, peu d'expériences impliquent les participants dans l'ingénierie même du processus participatif, ainsi que dans son pilotage.

\section{Du point de vue de la recherche}

Dans la recherche française, l'ingénierie de la participation est abordée à travers des travaux sur les ingénieurs de la participation (ex. : Mazeaud, 2012), les procédures participatives, outils et dispositifs (ex: Bonaccorsi et Nonjon, 2012) ou encore les idées, savoirs et expertises portés par ces ingénieurs via ces machineries (ex. : Crémin et al., 2018).

D'autres travaux portent sur la conception participative de projets, produits ou services, dans lesquels la participation se situe bien en amont du cycle décisionnel ou du cycle projet, mais dont l'objet n'est pas le processus participatif. Ce sont par exemple des travaux sur la recherche participative (Contamin et al., 2017), des réflexions sur les sciences de la participation (Blatrix, 2012), des recherches sur la démarche de co-conception d'un produit ou d'un service (Debos et al., 2017) ou encore sur la conception participative de projet. La co-conception de processus ou d'outils décisionnels («participatory design») s'inscrit dans la lignée de travaux sur la conception des systèmes décisionnels avec leurs utilisateurs (Kensing et Blomberg, 1998 ; Kujala, 2003 ; Vink et al., 2008; Jakku et Thorburn, 2010 ; Valls-Donderis et al., 2013). Dans notre cas cependant, les étapes, les rôles et les règles du dispositif futur de participation sont conçus par les participants eux-mêmes, auxquels il est donc demandé un exercice réflexif de projection.

Dans la littérature anglo-saxonne, Bryson et al. (2012) ont effectué une revue systématique de 250 articles et livres afin d'identifier 12 recommandations pour l'ingénierie, le pilotage et l'évaluation de processus participatifs en vue d'atteindre les résultats souhaités. Ils précisent que ces recommandations ne constituent pas une recette ni des règles à suivre dans la mesure où l'ingénierie participative doit être itérative, et ajustée en fonction d'objectifs et de contextes spécifiques. Ils pointent ainsi la principale controverse qui sous-tend la notion d'ingénierie de la participation et en nourrit les critiques : dès lors que l'on fournit un cadre au design ou à l'ingénierie de la participation, ne risque-t-on pas d'orienter, voire de manipuler le processus participatif? Cette controverse fait écho à celle mise en avant par 
l'institutionnalisme critique, qui remet en cause le principe de design institutionnel (Cleaver et Franks, 2005 ; Hassenforder et al., 2015).

Ainsi, l'ingénierie de la participation, de par les décisions stratégiques qu'elle présuppose, est «loin d'être neutre et transparente» (Bonaccorsi et Nonjon, 2012). Comme dans tout domaine technique, l'intervention d'intermédiaires professionnels induit un risque de contrôle de la participation par des personnes ayant des compétences en sciences sociales et considérées de par ce savoir comme légitimes pour gérer les participants (Blondiaux, 2008). Elle peut également entraîner des risques de capture politique du dispositif (Williams, 2004) ou encore une réduction de la participation aux outils et prestations proposées (Bonaccorsi et Nonjon, 2012). Elle détermine un accès différencié à des étapes décisionnelles dont la portée politique et technique est variable : état des lieux, cadrage politique, options, choix, mise en œuvre.

Notre hypothèse repose donc sur l'idée qu'associer des citoyens à l'ingénierie même de la participation diminuerait ces risques de manipulation et de remise en question du dispositif dans la mesure où les règles, les valeurs et les choix sous-tendant la démarche participative seraient coécrits par les acteurs concernés.

\section{L'expérience du bassin versant de la Drôme (2016-2017)}

\section{Contexte}

En matière de gestion intégrée des ressources en eau (GIRE), un des principaux instruments en France est le schéma d'aménagement et de gestion des eaux (SAGE). Il est élaboré au niveau d'un bassin versant par une commission locale de l'eau (CLE). Le schéma fixe les objectifs généraux d'utilisation, de mise en valeur et de protection quantitative et qualitative des ressources en eau superficielle et souterraine. La CLE est une assemblée délibérante, indépendante et décentralisée, chargée de la préparation et de la mise en œuvre du SAGE. Les citoyens ne participent pas directement à cette commission mais ils y sont représentés par le biais des élus des collectivités locales et par des représentants des associations d'usagers, des propriétaires fonciers ou des organisations professionnelles.

Sur le bassin versant de la rivière Drôme, à l'est de Valence (Fig. 1), la CLE est composée, au moment du projet, de 48 membres dont 27 représentants des collectivités territoriales et 16 représentants d'usagers. Ce bassin versant, de $1640 \mathrm{~km}^{2}$ et 50000 habitants, est le premier en France à avoir mis en place un SAGE dès 1997, doublé d'un second contrat de rivière (1999-2006), faisant suite à un premier contrat de rivière (1990-1996). Depuis 2007, la maîtrise d'ouvrage de l'animation du

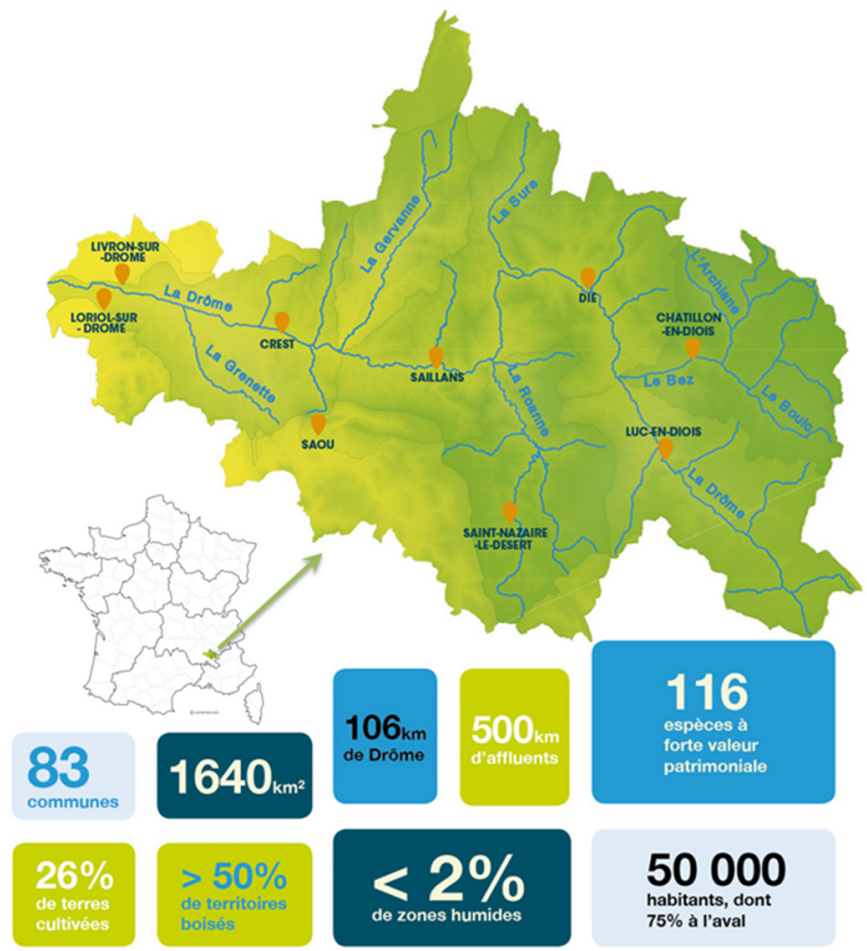

Fig. 1. Le bassin versant de la Drôme (source: SMRD).

schéma d'aménagement est portée par le syndicat mixte de la rivière Drôme et de ses affluents (SMRD). Le SAGE a été révisé une première fois entre 2007 et 2013 et devait l'être à nouveau à partir de 2018 (Girard, 2012; Girard et Rivière-Honegger, 2014). Le premier schéma d'aménagement et de gestion des eaux (2007) traitait essentiellement des questions de partage et d'économie de la ressource en eau notamment pour l'irrigation, l'aménagement physique de cours d'eau pour lutter contre l'incision du lit et des berges de la rivière et la reconquête de la qualité de l'eau. Celui de 2013 abordait trois enjeux supplémentaires : la préservation et la mise en valeur des zones humides, la délimitation d'un espace fonctionnel pour la gestion physique des cours d'eau et l'accueil des activités touristiques et de loisirs tout en préservant les milieux. Pour préparer la nouvelle révision, le syndicat mixte a souhaité élargir la participation au-delà des membres de la commission locale de l'eau en permettant à des citoyens de faire des propositions, en amont de la procédure institutionnelle de révision du SAGE, sur la période 2016-2018. Dans la poursuite des coopérations existantes depuis plus de vingt ans entre des chercheurs et des acteurs sur ce territoire, l'Irstea (Institut national de recherche en sciences et technologies pour l'environnement et l'agriculture $)^{6}$ est venu en appui du SMRD pour la réalisation de cet objectif, dans le cadre du projet SPARE.

6 iInstitut devenu INRAE après la fusion d'Irstea et de l'Inra en 2020. 


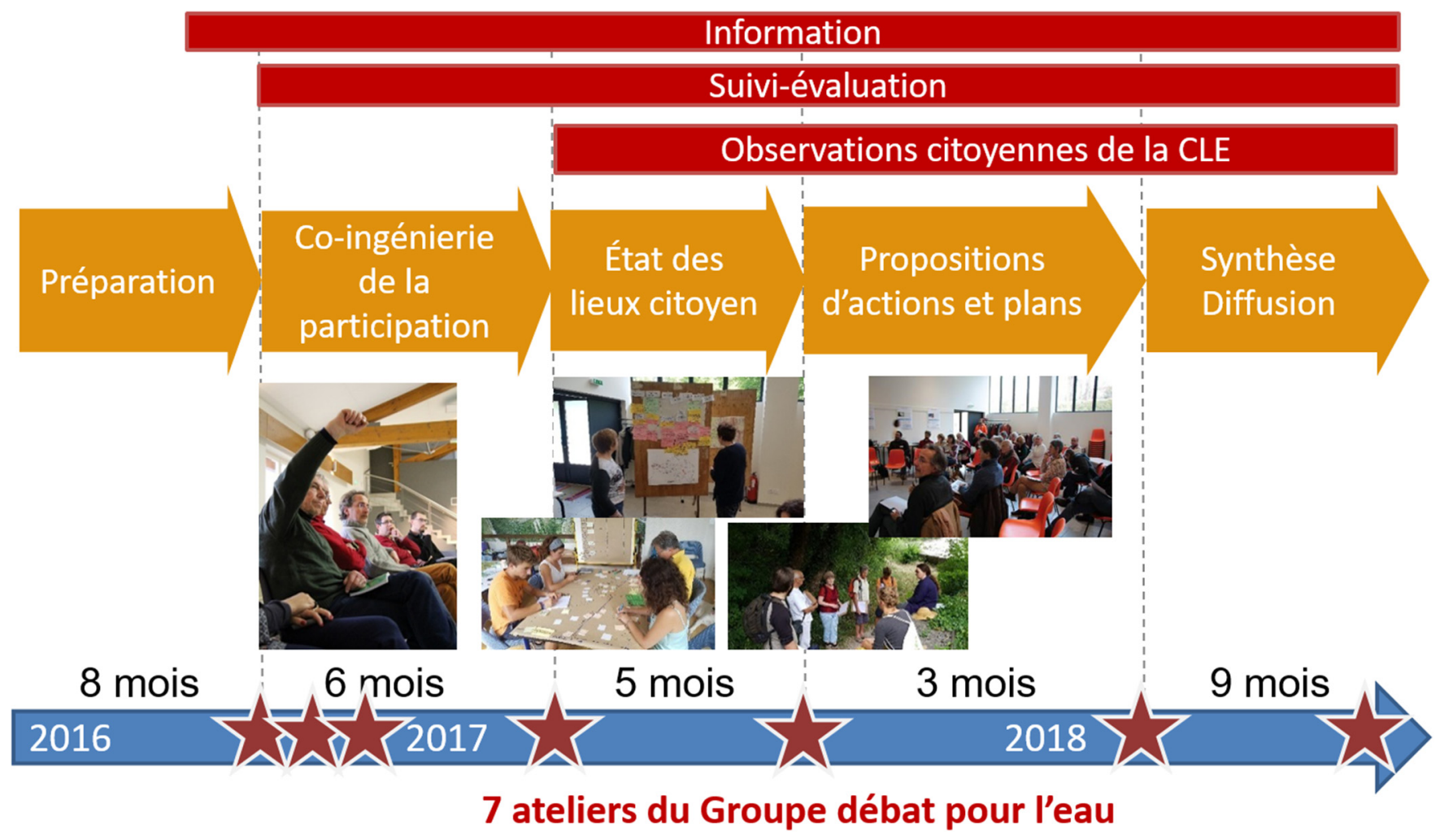

Fig. 2. Les étapes de la démarche participative sur la Drôme (Source: SMRD et Irstea).

La démarche participative a duré presque trois ans et incluait plusieurs étapes (Fig. 2). La préparation de la démarche incluait la constitution et les premières réunions du Groupe Pilote et les réunions d'information du public. L'étape de co-ingénierie de la participation s'est déroulée de décembre 2016 à mai 2017. Elle a été suivie par une troisième étape d'état des lieux citoyen puis par une quatrième étape de propositions d'actions et de plans. Enfin, la dernière étape consistait à produire la synthèse des données, à transmettre les résultats à la CLE et à promouvoir l'expérience. Les étapes 3, 4 et 5 ont été définies au cours de l'étape de co-ingénierie.

\section{La co-ingénierie de la participation dans le cas de la Drôme}

Plusieurs acteurs ont contribué à la co-ingénierie de la démarche participative (Annexe $1^{7}$ et Fig. 3). La directrice du syndicat mixte en était l'instigatrice, avec l'appui de son président. Elle était aussi la manager de la démarche: elle assurait la mise en œuvre opérationnelle. Une facilitatrice a été embauchée par le SMRD en avril 2016 afin d'organiser, d'animer et de faciliter tous les évènements participatifs avec les différentes parties prenantes. Le syndicat a fait appel aux chercheurs d'Irstea pour l'accompagner dans la conception, la mise

\footnotetext{
$\overline{7}$ Pour consulter les annexes, voir dans la section «Matériel supplémentaire ».
}

en œuvre et le suivi-évaluation de la démarche participative, ainsi que dans l'analyse des données. Trois chercheurs ont ainsi participé à la plupart des évènements participatifs ${ }^{8}$. Un groupe pilote, composé de 10 habitants bénévoles, dont 4 membres de la CLE, a été constitué par la manager et la facilitatrice en avril 2016 afin de soutenir le SMRD dans ses choix stratégiques visà-vis du dispositif participatif. Les critères de sélection étaient des personnes de confiance pour la manager, représentant dans la mesure du possible les principaux groupes et secteurs sociaux du territoire, ouvertes et intéressées par la participation. Les membres du groupe pilote participaient également aux réunions du groupe Débat pour l'eau, qui est l'acteur central de l'ingénierie de la participation. Le groupe Débat pour l'eau était ouvert, c'est-à-dire que toute personne pouvait l'intégrer en participant à l'une de ses réunions. Il était composé de 46 personnes, pour la plupart des citoyens habitant le bassin versant de la Drôme. Le parti pris de la démarche était de ne pas catégoriser les citoyens en fonction de l'enjeu autour de l'eau qu'ils portaient. Ainsi, il n'a jamais été demandé aux participants d'exprimer s'ils étaient agriculteurs, hôteliers, pêcheurs ou loueurs de canoës. Ils étaient invités en tant que citoyens, justement pour ne pas avoir à représenter un enjeu spécifique et pouvoir s'exprimer sur divers enjeux. L'annexe 1 inclut

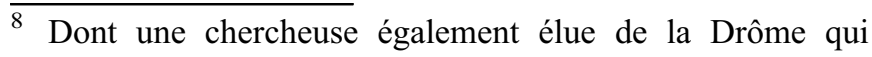
participe, à ce titre, aux réunions de la CLE. 


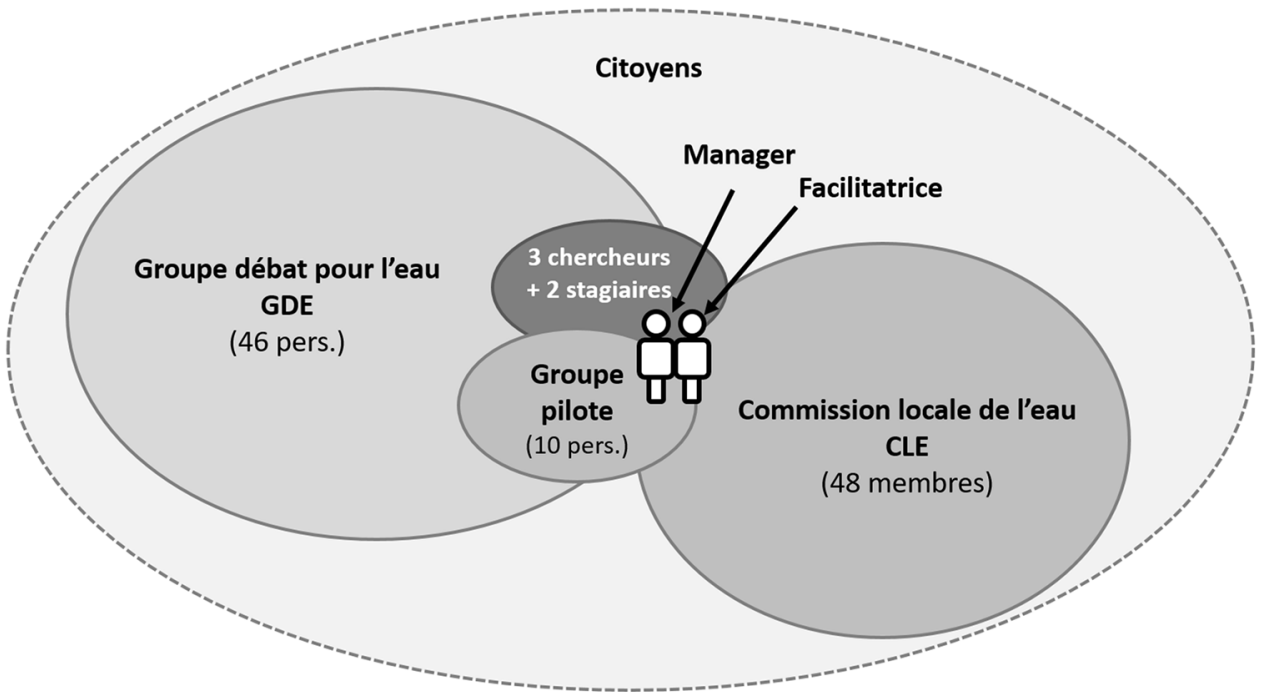

Fig. 3. Les acteurs ayant contribué à la co-ingénierie de la participation (Source : auteurs).

\section{Encadré 1. L'outil PrePar (Préparer la participation)}

L'outil PrePar fait partie de l'approche «CoOPLAGE ${ }^{9}$ » développée par les chercheurs d'Irstea (Ferrand et al., 2021 ; Hassenforder et al., 2021a). PrePar amène les participants à expliciter les attendus, les étapes et actions participatives, les rôles précis des différents acteurs en leur sein, ainsi éventuellement que les méthodes participatives envisagées. Cela précise le «niveau» effectif et la forme des différentes participations d'acteurs au processus décisionnel.

La méthodologie PrePar inclut six phases (cf. Annexe 2 et Ferrand et Noury, 2017):

1 Formaliser les objectifs de gestion et de participation

2 Identifier les parties concernées (participants potentiels)

3 Valider les étapes du processus décisionnel

4 Lister les actions de participation

5 Définir le rôle des parties prenantes pour chaque action, selon une typologie

6 Discuter les méthodes participatives

Le résultat produit est un plan de participation (Annexe 3). Les étapes décisionnelles sont issues des quatre phases du processus décisionnel identifiées par Simon (1977) et ont été détaillées et adaptées afin de correspondre au processus décisionnel «standard» de la gestion de l'eau en France (Annexe 4). Elles sont complétées par des «actions» qui les instancient de façon plus précise. Ainsi, par exemple, une étape d'état des lieux participatif avec des citoyens peut inclure trois actions complémentaires: une visite de terrain, une cartographie sensible à domicile et une session en salle de modélisation participative enjeux - acteurs - activités - ressources.

la composition du groupe Débat pour l'eau en termes de genre, d'âge et de localisation sur le bassin versant (amont, milieu de bassin ou aval). Ces personnes ont été mobilisées via divers canaux de communication (affiches, lettres d'information, interviews pour les radios locales, articles de presse) et dans le cadre de trois réunions d'information en novembre 2016. Le président de la CLE est intervenu de manière ponctuelle dans les réunions de ce groupe. Les citoyens du bassin versant étaient informés de la démarche par divers canaux de communication et par les différents acteurs impliqués.

\footnotetext{
${ }^{9}$ Coupler des outils ouverts et participatifs pour laisser les acteurs s'adapter pour la gestion de l'eau.
}

La co-ingénierie de la participation a duré six mois. Les trois principaux évènements clés sont les trois ateliers du groupe Débat pour l'eau respectivement en décembre 2016, février et mars 2017 (Tab. 1). Le cadre méthodologique mobilisé pour structurer cette coingénierie de la participation est l'outil PrePar, présenté dans l'encadré 1 . Ces ateliers étaient animés par la facilitatrice. Ils alternaient des moments en plénière, des travaux de groupe et des réflexions individuelles, sur la base de supports d'information et de travail préparés avec l'appui des chercheurs.

Le premier atelier participatif a eu lieu le samedi 3 et le dimanche 4 décembre 2016 et a rassemblé 32 citoyens sur les deux jours. Lors de ce premier atelier, les participants ont formalisé les objectifs de participation et 
Tab 1. Principales étapes de la préparation et de la co-ingénierie de la participation Drôme.

\begin{tabular}{|c|c|c|}
\hline Étape & Dates & Nom de l'évènement \\
\hline \multirow{5}{*}{ Préparation } & Avril 2016 & Recrutement du facilitateur et du groupe pilote \\
\hline & 7 juin 2016 & $1^{\mathrm{re}}$ réunion du groupe pilote \\
\hline & 11 juillet 2016 & $2^{\mathrm{e}}$ réunion du groupe pilote \\
\hline & 5 septembre 2016 & $3^{\mathrm{e}}$ réunion du groupe pilote \\
\hline & 15 et 19 novembre 2016 & Réunions d'information \\
\hline \multirow{8}{*}{ Co-ingénierie } & 3 et 4 décembre 2016 & $1^{\mathrm{er}}$ atelier du groupe Débat pour l'eau \\
\hline & 16 janvier 2017 & $4^{\mathrm{e}}$ réunion du groupe pilote \\
\hline & 6 février 2017 & $2^{\mathrm{e}}$ atelier du groupe Débat pour l'eau \\
\hline & 6 mars 2017 & $3^{\mathrm{e}}$ atelier du groupe Débat pour l'eau \\
\hline & 16 mars 2017 & Approbation de la charte par la CLE \\
\hline & 31 mars-17 avril 2017 & $\begin{array}{l}\text { Consultation en ligne sur le } \\
\text { plan et les règles de participation }\end{array}$ \\
\hline & 24 avril 2017 & $1^{\mathrm{er}}$ atelier de suivi-évaluation participatif \\
\hline & 13 mai 2017 & Atelier de lancement du dispositif participatif \\
\hline
\end{tabular}

identifié les parties prenantes qui seraient impliquées dans les étapes ultérieures de la démarche participative. Pour cela ils disposaient comme support de débat des «fiches étapes» leur permettant de choisir la pertinence des étapes et l'intensité de la participation citoyenne pour chacune $^{10}$. Les participants ont également identifié les actions de participation à mettre en œuvre, par exemple instituer le groupe Débat pour l'eau, rendre compte régulièrement aux acteurs du territoire, définir une charte de la participation ou encore créer des espaces d'écoute des citoyens hors des lieux habituels. Une première ébauche de plan de participation a ainsi été réalisée. Dans la mesure où les participants n'avaient pas eu le temps de découvrir les méthodes participatives possibles pour chaque étape, l'Irstea a proposé que le choix de ces

\footnotetext{
${ }^{10}$ Ces fiches étapes sont disponibles au lien suivant: https:// www.eaurmc.fr/jcms/pro_89960/fr/quelle-strategie-participa tive-pour-la-gestion-locale-de-l-eau-avec-les-citoyensvolume-2-fiches-etapes. Elles ont été produites dans le cadre d'un projet piloté par l'Irstea et l'Agence de l'Eau Rhône Méditerranée Corse intitulé "Quelle stratégie participative pour la gestion locale de l'eau avec les citoyens?» (20162020). L'ensemble des productions du projet sont disponibles au lien suivant: https://www.eaurmc.fr/joms/gbr 5509/fr/med iatheque cids $=\& \mathrm{cids}=\& \mathrm{cids}=\& \mathrm{cids}=\& \mathrm{cids}=\&$ tex $\mathrm{t}=\mathrm{strat} \%$ C3\%A9gie + participative \&OK=Rechercher.
}

méthodes revienne au groupe pilote. Par ailleurs, à l'issue de ce premier atelier, les participants ont exprimé leur volonté d'organiser un deuxième, puis un troisième atelier afin de permettre aux personnes qui n'étaient pas disponibles lors des journées de décembre de contribuer à l'élaboration du plan de participation. Entre-temps, le groupe pilote et les chercheurs ont suggéré une trame de fiches à remplir pour expliciter les différentes actions de participation. Ils ont également fait des suggestions sur les méthodes participatives qui pourraient être utilisées (jeux de rôles, prospective participative, etc.) et sur le suivi-évaluation de la démarche. Lors des ateliers du 6 février et du 6 mars 2017, les participants ont ainsi complété le plan de participation en remplissant des fiches-action comme celle présentée dans la figure 4.

Lors de l'atelier du 6 mars, les participants se sont interrogés sur leur légitimité à «décider de qui allait participer ». Ils ont décidé de réaliser une consultation en ligne permettant à tous les membres du groupe Débat pour l'eau de donner leur avis sur le plan de participation produit au préalable. Huit avis ont été enregistrés, dont sept validant les documents ${ }^{11}$. Le plan final (Fig. 5) a été

\footnotetext{
11 Une des réponses s'opposait à la validation en l'état des documents en argumentant que le prochain atelier du GDE avait justement pour objet de les valider. Une réponse à été faite en expliquant qu'il s'agissait d'un malentendu.
} 

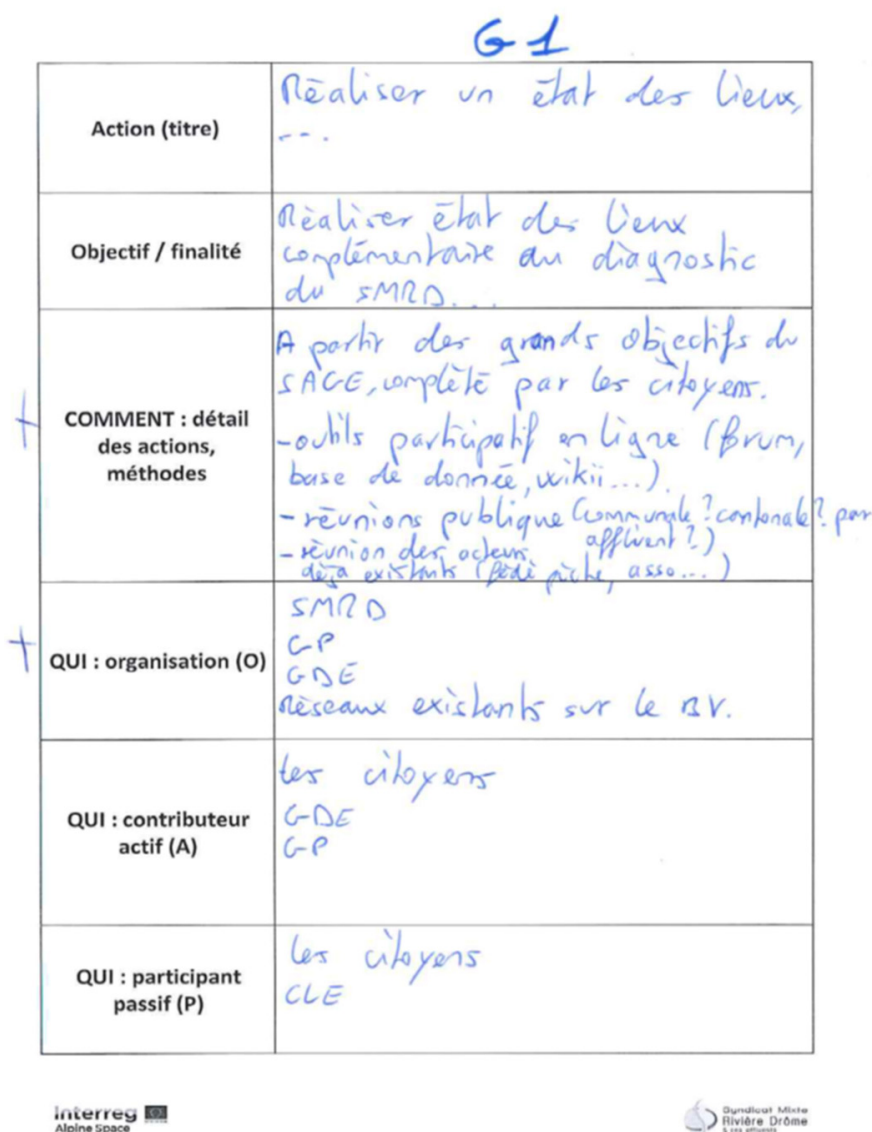

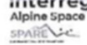

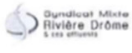

Fig. 4. Aperçu d'une fiche «action de participation» complétée par les participants le 6 février 2017.

présenté publiquement le 13 mai 2017 lors de l'atelier de lancement $\mathrm{du}$ dispositif participatif, qui a marqué également le début de la mise en œuvre du plan de participation, en commençant par un état des lieux citoyen.

Par ailleurs, la manager et la facilitatrice du syndicat mixte de la rivière Drôme ont élaboré des règles de la participation, appuyées par les chercheurs. Ces règles n'ont pas été élaborées de façon participative, car cela aurait demandé un nouvel investissement des citoyens, alors que ces derniers étaient déjà mobilisés sur la conception du plan de participation. Ces règles ont donc été conçues par des experts ${ }^{2}$. Toutefois, elles ont été soumises à discussion et ajustées en fonction des retours des participants lors des réunions des 6 février et 6 mars 2017, puis soumises à consultation au même titre que le

\footnotetext{
${ }^{12}$ Ces règles ont été élaborées sur la base de la charte de la participation du public portée par le ministère de l'Environnement, de l'Énergie et de la Mer qui venait tout juste d'être publiée (cf. https://www.ecologique-solidaire.gouv.fr/charteparticipation-du-public). Tous les documents sont disponibles sur le site internet du SMRD : https://www.riviere-drome.fr/ actions-etudes/projet-spare.
}

plan de participation. Ces règles, construites sur le modèle du règlement intérieur de la CLE, avaient pour objectif de formaliser l'existence des différents groupes participatifs et également de réguler leur fonctionnement. Elles incluaient des éléments sur la composition du groupe pilote et du groupe Débat pour l'eau, leurs rôles, la fréquence des rencontres, les modalités de validation des décisions et les engagements.

La manager et la facilitatrice du SMRD ont également rédigé une charte pour formaliser les relations entre le groupe institutionnel de gestion de l'eau (la CLE) et le groupe citoyen (groupe Débat pour l'eau). La charte assurait la reconnaissance du projet SPARE et des groupes citoyens ainsi que la prise en compte des travaux du groupe Débat pour l'eau par la commission locale de l'eau. Elle permettait également à des citoyens volontaires d'être observateurs aux réunions de la CLE.

Enfin le suivi-évaluation de ce processus d'ingénierie de la participation a été réalisé grâce au cadre méthodologique ENCORE-MEPPP ${ }^{13}$ (Hassenforder et al., 2016). Différentes méthodes ont été mobilisées: questionnaires fermés, listes de présence, expression des attentes, observation participante, carnet de bord, photos et vidéos, entretiens semi-directifs (Annexe 5). Les résultats présentés sont issus de l'analyse de l'ensemble de ces données, en particulier des entretiens semidirectifs (Annexe 6). Un groupe citoyen spécifique a participé au cadrage du suivi-évaluation.

Ainsi, cette phase de co-ingénierie de la participation devait concilier plusieurs contraintes: la taille et la représentativité du groupe présent, les expériences ou connaissances variées sur la participation, la surprise ou le dépit de ne parler «que » de la participation à ce stade, le temps et la disponibilité des personnes, l'accompagnement par un projet de recherche-intervention ayant son propre agenda. L'organisation pratique s'est appuyée sur différentes instances et sur la combinaison de travail individuel et de groupes mixés, des réflexions à domicile et des supports d'orientation sur les actions et la procédure (ex. fiches), créant ainsi un cadre où développer les contributions. Des critiques ont porté précisément sur l'excès ou, au contraire, le manque de cadrage.

13 MEPPP est l'acronyme de «monitoring and evaluating participatory planning processes» (suivre et évaluer des processus de planification participative). La démarche détaille différentes étapes à suivre pour construire un processus de suivi-évaluation d'une démarche participative. Il est ainsi possible de suivre et d'évaluer différents types d'impacts. ENCORE propose six catégories d'impacts : externe, normatif, cognitif, opérationnel, relationnel, équité. 


\section{Plan de participation bassin Drôme}

\section{7 ÉTAPES}
Mars Préparation - Cadrage du plan de participation; charte ; règles et principes de de la participation
- Publication du plan, récolte des avis, consolidation

Avr.

Forum de

lancement pour les étapes à venir
Présentation du plan validé et choix pour chaque participant de son rôle

- Cartographie participative en ligne

- Enquête en ligne et porte à porte,

- Porteurs de parole et relais sur tout le bassin,

- Wiki \& Supports pédagogiques

État des - Echanges participants - experts

lieux / - Observation participative sur le terrain avec le Kit « ROCK »

diagnostic/ - Modélisation participative (Jeu de rôles Wat-A-Game)

glossaire - Petit jeu permettant de cartographier le bassin versant

- Glossaire avec une section « Foire Aux Question » (FAQ) ou « j'y comprends rien... »

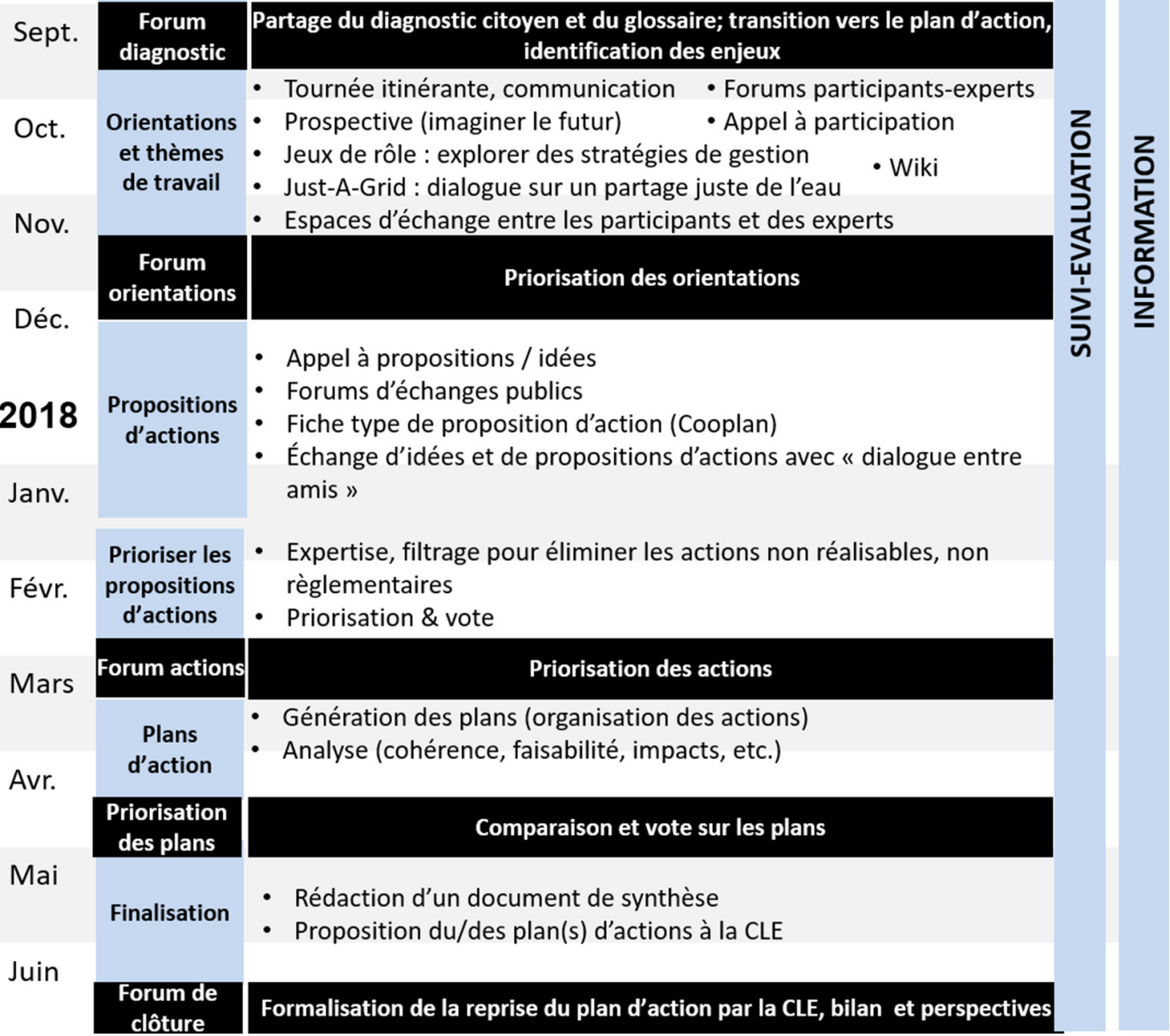

Fig. 5. Plan de participation du bassin versant de la Drôme (Source: SMRD et Irstea). 


\section{Apports et limites d'une co-ingénierie de la participation}

La question qui nous intéresse est: qu'apporte aux participants et au processus l'implication des citoyens dès l'étape d'ingénierie de la participation? Nous nous concentrons dans cette analyse sur les retours d'expérience concernant la co-ingénierie de la participation de manière générale en laissant de côté les retours concernant l'outil PrePar spécifiquement. Cette analyse peut s'appuyer sur deux familles de critères : des critères exogènes définis ex ante par les instigateurs ou les chercheurs, et des critères endogènes au processus et codéfinis par le groupe participatif «suivi-évaluation" évoqué ci-dessus. Quoiqu'ils puissent se recouvrir, il est probable que la satisfaction à l'égard de la co-ingénierie soit plus forte en ce qui concerne les critères que les participants ont eux-mêmes définis. En parallèle, des critères plus généraux permettent une évaluation comparative plus large. Dès lors, nous discutons ici des critères définis ex ante par les pilotes gestionnaires. Le dispositif est-il plus adapté aux attentes des citoyens, est-il mieux compris, approprié? Les citoyens sont-ils davantage engagés dans la suite du processus, sont-ils plus aptes à participer? Enfin, la co-ingénierie amélioret-elle l'articulation entre le dispositif participatif et le processus de décision institutionnelle?

\section{Appropriation citoyenne de cette co-ingénierie et du processus ultérieur}

Faire venir et faire rester les citoyens pour parler d'abord de participation et non de la rivière a été un défi. En effet, pour beaucoup, le facteur déclenchant leur mobilisation est l'intérêt porté à l'eau ou à la rivière. Rapidement, plusieurs participants ont perçu l'étape initiale de co-ingénierie de la participation comme trop abstraite et éloignée de leurs préoccupations. Ils ont remis en cause l'intérêt de co-construire le plan de participation. Parmi ces participants, certains considéraient que l'ingénierie devrait être confiée à des spécialistes, tels que les chercheurs ou le gestionnaire: « la préparation de la participation, je dis qu'il n'y a que les gens qui ont travaillé là-dessus, qui sont techniciens, qui peuvent informer et guider les gens d'une certaine manière, après quand c'est mis en route, on peut faire participer tout le monde» $\left(\mathrm{C}^{14}\right)$. Pour d'autres, la participation des citoyens devait se concentrer sur des thèmes déjà définis et sur des projets concrets, l'ingénierie leur apparaissant comme un travail trop théorique : «Le premier forum a dû en fatiguer beaucoup parce que cogiter pour savoir comment on va cogiter, il

\footnotetext{
${ }^{14}$ Les codes entre parenthèses font référence au codage des entretiens (Annexe 7).
}

faut déjà être bien accroché pour l'avaler» $(\mathrm{C} 1)$. Ces difficultés ont amené plusieurs participants à quitter le processus : un tiers des participants (18/46 présents à ce moment) n'ont pas participé à la mise en œuvre du plan de participation par la suite. Néanmoins ceux qui sont restés, ainsi que le maître d'ouvrage et certains élus de la CLE, ont pris conscience des enjeux liés à l'étape d'ingénierie pour la suite du processus. Un citoyen a mentionné, à propos de l'ingénierie de la participation: «C'est sûr que ce serait plus simple pour nous [...] si on avait déjà la méthode, les phases, le planning et les supports avec les bonnes questions. Mais ce serait moins intéressant et puis ce serait peut-être moins bien parce qu'on ne se poserait peut-être pas les bonnes questions » (C3). Un élu s'est exprimé, à propos de l'étape d'ingénierie: «elle ralentit le processus, mais elle fait gagner du temps pour après; [...] c'est mieux de le faire avec les citoyens, parce qu'au moins ils ont l'impression de vraiment être investis à fond » (E1).

L'appropriation du processus participatif par les citoyens à travers leur implication dans la co-ingénierie s'est traduite par deux aspects. D'une part, les participants ont accordé une grande importance à la transparence et à l'exactitude des comptes rendus, en relisant méthodiquement les documents produits par le gestionnaire. D'autre part, ils ont remis en cause certains éléments de la méthode participative proposée par l'Irstea. Ainsi, un participant a indiqué : "Les grands tableaux, c'est vrai qu'à des moments ils étaient difficiles à comprendre [...] il y avait beaucoup de choses... il faudrait qu'ils soient simplifiés, parce qu'après on a l'impression qu'on fait un tableau pour faire un tableau » (C4). Ce qui est important ici n'est pas le contenu des critiques que les participants ont émises, qui sont propres à PrePar et peu généralisables, mais le fait même que les citoyens aient porté un regard critique sur les méthodes participatives proposées. Cela témoigne d'une appropriation critique de la démarche participative. Enfin, dans l'élaboration du plan, les citoyens ont choisi de ne pas investir certaines étapes ou méthodes, qui leur sont finalement apparues comme nécessaires. Les chercheurs et les managers ont assumé les conséquences de ce plan et proposé aux citoyens de l'adapter chemin faisant, au gré de l'apparition des besoins et des critiques. La coingénierie s'est ainsi poursuivie au-delà de la phase de préparation, amenant l'ensemble de parties prenantes à se responsabiliser sur son contenu.

\section{Traduction et recherche d'informations}

L'étape de co-ingénierie de la participation a permis de mettre d'accord les différents acteurs sur un langage et un vocabulaire communs. Le 3 décembre 2016, un participant a mentionné « j'ai failli pas venir parce que dans la presse on disait 'gestion de l'eau' et ça c'est une 
conception d'hydraulicien, de tuyaux » (C3). Les discussions ont permis de mettre à jour des incompréhensions, portant tant sur les informations et les données techniques liées à la gestion de l'eau que sur le vocabulaire de la participation, puis de les clarifier. Néanmoins, il ne faut pas négliger que l'étape d'ingénierie est un exercice difficile intellectuellement car beaucoup de participants n'ont pas l'habitude de manier des notions abstraites et techniques, ou de travailler collectivement en réunions, d'autant plus lorsqu'elles se tiennent sur tout un week-end. Un participant témoigne: «Il y avait instit, il y avait chercheur, il y avait machin. Que des gens qui ont plus l'habitude de manier des concepts intellectuels. [...] Alors que ce n'est pas à mon avis la façon qu'a l'habitude de penser et de travailler la majorité des gens. Il aurait fallu que ce soit plus [...] terre à terre » (E2). Ce déficit de traduction a été pointé par un autre participant: «on a tous dans notre boulot un jargon professionnel, ça fait gagner du temps, mais normalement quand on parle à des profanes, on traduit en français courant» (C2). L'appropriation et la participation active nécessitent en effet de la traduction, c'est-à-dire de trouver un langage commun entre les différents acteurs et de laisser s'exprimer différents points de vue et de la controverse (Blanc, 2011). Dans le cas de la Drôme, la facilitatrice a joué un rôle central dans cette traduction. Sa connaissance à la fois des aspects techniques de la gestion de l'eau (car étant au préalable chercheuse puis consultante dans ce domaine) et du vocabulaire de la participation (suite à plusieurs formations dans le cadre du projet SPARE) lui ont permis d'être coutumière de ces différents langages et ont facilité son rôle de traductrice.

En parallèle, ce besoin de traduction a amené plusieurs participants, entre deux réunions, à aller chercher de l'information sur la gestion des rivières pour appuyer leurs choix, dès cette phase de co-ingénierie puis tout au long du dispositif participatif. En effet, la manager avait choisi de ne pas prédiffuser les documents administratifs, comme les plans antérieurs, pour ne pas influencer le contenu. Des citoyens ont cherché des rapports existants ou produit des synthèses écrites sur la gouvernance de la rivière Drôme ou sur des aspects techniques, qu'ils ont communiqués aux autres participants. S'il est difficile d'attribuer la causalité absolue de l'ingénierie de la participation à ces initiatives, elle y a tout du moins contribué. Cela fait écho aux observations de Marion Carrel sur plusieurs ingénieries participatives qui avaient pour principal effet de susciter l'intérêt du public par la coproduction d'une enquête sur une thématique ou sur le fonctionnement des institutions (Carrel, 2007).

\section{Régulation sociale}

Pour disposer d'un cadre dès le départ, des règles de participation ont été élaborées par la manager et la facilitatrice sur la base de documents similaires produits par d'autres collectivités. La co-ingénierie ultérieure a permis aux participants de les discuter et de se les approprier. Ainsi, une forme de régulation sociale était opérationnelle dès le démarrage du processus participatif lui-même. Cet aspect a été synthétisé par une des personnes interrogées: "En fait, cadrer, ça veut dire respecter les règles et remettre les gens dans le respect des règles. Quand on ne se connaît pas, si ce n'est pas fait par un tiers, c'est impossible. Par contre, quand on arrive à être dans des groupes suffisamment homogènes, où tout le monde accepte les règles, même s'il les enfreint parfois, quand le reste du groupe fait remarquer qu'il dévie, qu'il l'accepte, on n'a pas besoin d'une tierce personne pour faire ça» $(\mathrm{C} 3)$.

Cependant, le rôle clé du facilitateur s'est révélé, notamment au début du processus, dans la gestion de la dynamique de groupe et des disparités entre les participants. Deux limites à cette régulation sociale sont néanmoins apparues en cours de processus. Premièrement, ce type de régulation est moins efficace dans un groupe ouvert qu'au sein d'un groupe fermé, par définition plus stable dans le temps, et dans lequel, d'une part les participants apprennent à se connaître et $\mathrm{d}$ 'autre part, définissent, formellement ou pas, leurs propres règles de fonctionnement. Le fait que le groupe Débat pour l'eau restait ouvert et accueillait constamment de nouveaux participants nécessitait un temps d'appropriation des règles, du travail déjà réalisé par les autres participants et un apprentissage social ralentissant cette régulation. Les nouveaux participants devaient en arrivant valider les documents préexistants (chartes, règles, plan de participation...). Deuxièmement, les règles de participation étaient initialement conçues pour les échanges en présentiel. Elles se sont révélées comme insuffisamment adaptées aux échanges dématérialisés (emails et forum) et n'ont pas toujours permis de contenir les prises et les abus de pouvoirs. Des règles de modération spécifiques (numériques) ont été mises en place dans un deuxième temps et le gestionnaire a dû recadrer les échanges, ce qui a donné lieu notamment à l'exclusion d'un participant.

\section{Apprentissages sociaux et sentiment d'appartenance au groupe}

Cette régulation est renforcée par des apprentissages sociaux et un sentiment d'appartenance au groupe qui interviennent en amont du dispositif. Ainsi, lorsque les citoyens commencent à parler de la rivière lors de l'étape d'état des lieux, le collectif est fonctionnel, «la dynamique de groupe nécessaire existe» $(\mathrm{O} 1)$ et un sentiment de confiance s'établit entre les participants, ce qui prédispose à une participation plus efficace par la 
suite (Tippett et al., 2005). Un participant en témoigne : «Quand tu te prends au jeu [...] tu rentres dans quelque chose. [...] Après tu te sens lié aux gens. [...] Alors c'est chouette parce que si tu fais quelque chose avec eux, si tu deviens ami avec eux, si tu as des atomes crochus, tu es plus détendu dans ce que tu vas faire. [...] et peut-être que ça fera qu'on sera plus motivés que jamais » (C5).

Pendant l'étape suivante d'état des lieux, plusieurs évènements ont été auto-organisés par les participants, ce qui témoigne de leur autonomisation: réunions de discussion, ateliers participatifs lors d'évènements publics ou dans des écoles, sortie de terrain, etc. Cela s'est aussi traduit par des revendications collectives sur le fonctionnement (lieux de travail, maîtrise de perturbateurs, agenda). Le sentiment d'appartenance au groupe est renforcé par le fait que l'étape d'ingénierie de la participation implique que le groupe lui-même détermine son propre rôle dans les étapes décisionnelles à venir, ce qui le force à se positionner collectivement. Mais on peut se demander si ce travail de construction du collectif ne serait pas plus efficace si les citoyens avaient également construit les règles de participation. Il semble en effet que la co-définition des règles ait ses effets propres. Une telle élaboration préliminaire nécessiterait des participants une connaissance et une réflexion sur les conditions et les risques de la participation qui seraient réservées à une minorité déjà expérimentée. L'apport exogène semble donc logique pour des «participants débutants », ainsi que le préconisent les institutions expertes comme l'Institut de la concertation et de la participation citoyenne.

Les différents éléments mentionnés ci-dessus (traduction, régulation sociale, apprentissages sociaux) rejoignent des observations effectuées par Philippe Lyet (2011) qui met en avant que l'activation du triptyque «sens (traduction)/régulation (transaction sociale)/ identité » acte la collaboration entre les participants et permet la construction d'un espace intermédiaire collaboratif grâce à un processus de traduction croisée.

\section{Articulation entre participation et gouvernance institutionnelle}

Une autre valeur ajoutée de la co-ingénierie de la participation est de permettre de clarifier l'articulation entre le processus décisionnel institutionnel et le processus participatif: d'une part cette articulation est explicitement abordée dans le processus de construction du plan de participation; d'autre part, le processus d'ingénierie de la participation agit comme un démonstrateur de l'engagement et de la capacité d'expression des citoyens pour des agents du syndicat de rivière et pour des élus de la CLE. Dans la Drôme, c'est pendant cette étape que la commission locale de l'eau a décidé, par le biais de l'approbation de la charte, de la prise en compte des avis des citoyens dans la révision institutionnelle du SAGE. Cela s'est traduit par l'inclusion des résultats de l'état des lieux citoyen dans la partie état des lieux du nouveau plan d'aménagement et de gestion durable du SAGE, et par l'inspection systématique des propositions d'actions citoyennes par les membres de la commission locale de l'eau lors des séances de travail pour la rédaction des objectifs généraux du nouveau schéma d'aménagement et de gestion des eaux. Cette validation a été un fort levier de motivation et d'engagement des participants car, ainsi, ils voyaient comment leurs contributions seraient considérées.

En parallèle, le fait de réfléchir aux objectifs de la participation a créé de la confusion chez les participants sur la marge de manœuvre réelle de leurs contributions dans le processus décisionnel, et ce, malgré le fait que le syndicat ait précisé dès le départ que la participation intervenait en amont de la révision du SAGE, et sans pouvoir légal. La discussion sur les objectifs a été déstabilisante pour plusieurs participants, sentiment accentué par la durée de cette étape: «j'ai eu l'impression que pendant des mois on ne savait pas où on allait»(C6). C'est finalement le gestionnaire et le groupe pilote qui ont défini et affirmé l'objectif au fil du processus. Ce retour d'expérience questionne l'intérêt de débattre des objectifs de la participation lorsque l'articulation de la participation avec la gouvernance institutionnelle est prédéfinie.

\section{Conclusion}

L'analyse de la co-ingénierie de la participation dans le cadre de la préparation de la révision du SAGE dans la Drôme présenté ici montre que celle-ci permet une appropriation de la démarche participative par les citoyens, que cela répond aux besoins de traduction et permet une forme de régulation sociale au sein du groupe ainsi que la construction d'un collectif. La co-ingénierie permet notamment que ces éléments soient rassemblés avant la mise en œuvre de la démarche participative, ce qui apparaît comme essentiel pour que la démarche soit plus transparente, qu'elle prenne mieux en compte les avis des citoyens et produise des changements plus effectifs. La co-ingénierie permet au groupe de participants de se connaître et de savoir comment fonctionner ensemble, avec des règles qu'ils ont euxmêmes définies, avant même de se lancer dans le vif du sujet, c'est-à-dire la révision du SAGE.

Certes, nous sommes conscients que certains éléments de cette expérience sont spécifiques à l'approche mobilisée (PrePar) et au territoire concerné (le bassin versant de la Drôme et ses acteurs). Notamment, parce que le bassin versant est un milieu rural dynamique en 
termes démographique, économique, social et culturel, et aussi parce que beaucoup d'élus ont eu la volonté d'adopter des approches plus participatives, ce qui a probablement favorisé la participation des habitants dans la démarche. La collaboration historique entre les chercheurs et les gestionnaires avec des relations de confiance établies a facilité la possibilité d'expérimenter la co-ingénierie. Enfin, la personnalité et les compétences de plusieurs des personnes clés de la démarche (la manager, la facilitatrice, la chercheuse également élue sur le territoire) ont été des facteurs essentiels dans sa mise en œuvre.

En parallèle, le fait que l'ingénierie de la participation soit un exercice assez théorique et abstrait, « cogiter pour savoir comment on va cogiter $»(\mathrm{C} 1)$ comme le dit un des participants, a amené plusieurs d'entre eux à quitter le processus et plus d'un tiers des participants (18/46) n'a pas participé à la mise en œuvre du plan de participation par la suite. Par ailleurs, cette mise en œuvre a été complexe pour la manager et la facilitatrice (manque de moyens humains et financiers, désengagement de certains citoyens par rapport aux idées qu'ils avaient eux-mêmes proposées, méthodes participatives proposées par les chercheurs plus visibles et soutenues que les autres, etc.). Cette complexité a fait que le plan de participation n'a pas pu être entièrement respecté.

Dans une perspective gestionnaire, ces limites posent la question d'un juste milieu qui pourrait peut-être être trouvé, permettant aux citoyens de contribuer à la coingénierie mais en réduisant l'ambition de la démarche sur plusieurs aspects ${ }^{15}$ :

- Limiter la durée de la phase de co-ingénierie : dans la Drôme, elle s'est déroulée en trois ateliers sur 6 mois. Si l'organisation d'ateliers supplémentaires visait l'élargissement du nombre de contributeurs (qui sont passés de 34 à 46), elle a aussi significativement prolongé la durée de la phase d'ingénierie et a nécessité de réexpliquer à chaque fois les avancées préalables, ce qui a créé une lassitude pour les participants réguliers.

- Centrer la co-ingénierie sur les décisions pour lesquelles les citoyens apportent la plus grande valeur ajoutée: 1) le choix de grandes étapes clés d'un plan de participation; 2) la sélection du niveau de participation à chaque étape qui leur permet de débattre de leur implication et des attentes vis-à-vis des étapes ultérieures, allant du recueil d'avis à la coconstruction; 3) l'identification des acteurs et de leur rôle à chaque étape décisionnelle car c'est par ce biais

\footnotetext{
15 Ces recommandations sont toutes issues des réflexions collectives menées dans le cadre du suivi-évaluation de la démarche, entre la manager, la facilitatrice, les chercheurs et les participants à la démarche (questionnaire d'évaluation, réunions dédiées, ...)
}

qu'ils peuvent décider de prendre une part active dans la suite du dispositif et identifier des vecteurs de mobilisation de la population, notamment en jouant un rôle d'intermédiaire et en activant leurs réseaux sociaux. Ces éléments ne sont pas propres à PrePar, on les retrouve dans la majorité des démarches d'ingénierie de la participation. D'autres choix peuvent au contraire rester entre les mains des seuls gestionnaires ou consultants : 1) l'opérationnalisation de la participation (choix des méthodes participatives, du calendrier, etc.) ; 2) le cadrage initial de l'objectif de la participation lorsque l'articulation de la participation avec la gouvernance institutionnelle est prédéfinie. Cela permet de clarifier en amont quelle est la décision en jeu (ici le SAGE), quels sont les mécanismes de prise de décision (par exemple, les réunions de la CLE), les acteurs de cette prise de décision et comment la participation s'articule avec ces éléments (modalités, temporalité, lieu, etc.).

- Permettre aux citoyens d'apporter un retour réflexif et de proposer des adaptations sur l'approche de coingénierie et sur le plan de participation au cours du dispositif. Cela pouvant se faire une fois que les participants ont vécu une première expérience concrète de participation, et qu'ils ont eu des interactions avec des professionnels et des élus, c'est-à-dire lorsqu'ils sont prêts à prendre part à la délibération. Ces constats ont également été mis en avant par M. Carrel (2007) quand elle évoque la «participation construite» dont les modalités et les principes sont proches de la coingénierie telle qu'elle est définie dans cet article.

Dans une perspective de recherche, des travaux ultérieurs nous semblent importants à mener sur les compétences des participants nécessaires à la coingénierie, sur l'intégration avec une transformation plus globale de la gouvernance et sur l'informatisation de l'accompagnement à la co-ingénierie. L'analyse des processus de traduction dans le sens down-top, c'est-àdire du langage vernaculaire vers les catégories expertes, nous semble également pertinente.

\section{Matériel supplémentaire}

Le matériel supplémentaire (Hassenforder et al., 2021b), constitué des 6 annexes listées ci-dessous, est accessible par ce lien : https://hal.inrae.fr/hal-03178457.

Annexe 1: Acteurs impliqués dans la co-ingénierie de la démarche participative de la Drôme.

Annexe 2: Les six phases pour concevoir un plan de participation (PrePar).

Annexe 3 : Matrice support pour la réalisation du plan de participation PrePar.

Annexe 4 : Correspondance entre les étapes de la décision proposées au sein de la méthodologie PrePar, les phases du 
processus décisionnel identifiées par Simon (1977) et les étapes standards d'élaboration du schéma d'aménagement et de gestion des eaux (SAGE).

Annexe 5 : Éléments quantitatifs sur le suivi-évaluation du dispositif participatif Drôme.

Annexe 6: Codage des entretiens.

\section{Remerciements}

Les auteurs remercient Bertrand Degueurce, membre du groupe pilote Drôme, pour ses commentaires et suggestions, et pour avoir apporté sa vision citoyenne à ce papier. Un grand merci revient également à tous les membres du groupe Débat pour l'eau et du groupe pilote de la Drôme pour leur énergie et leur engagement. Les auteurs remercient Florestan Groult, stagiaire Irstea en 2017, pour avoir mené des entretiens sur l'étape d'ingénierie. Les auteurs remercient également Alexis Ferrand pour sa relecture attentive de l'article.

Les auteurs remercient les organisateurs du $7^{\mathrm{e}}$ Colloque du réseau OPDE «Des outils pour décider ensemble» qui a eu lieu à Montpellier les 26 et 27 octobre 2017 et à l'occasion duquel une première version de ce texte a été présentée.

Enfin, les auteurs remercient les relecteurs ainsi que les éditeurs de Natures Sciences Sociétés pour leurs suggestions sur les versions antérieures de cet article.

Cette recherche a été cofinancée par l'Union européenne via le projet Interreg du programme Espace Alpin SPARE (http://www.alpine-space.eu/projects/ spare/). Elle ne reflète pas nécessairement les opinions du programme Espace alpin (PEA), qui n'est pas non plus responsable de tout usage qui pourrait être fait des informations contenues ici.

\section{Références}

Barbier R., 2005. Quand le public prend ses distances avec la participation, Natures Sciences Sociétés, 13, 3, 258-265, https://doi.org/10.1051/nss:2005040.

Bherer L., Gauthier M., Simard L. (Eds), 2017. The professionalization of public participation, London, Routledge.

Blanc M., 2011. Participation et médiation dans la recherche en sciences sociales : une perspective transactionnelle, Pensée plurielle, 28, 69-77, https://doi.org/10.3917/pp.028.0069.

Blatrix C., 2012. Des sciences de la participation: paysage participatif et marché des biens savants en France, Quaderni, 79, 59-80, https://doi.org/10.4000/quaderni.612.

Blondiaux L., 2008. Le nouvel esprit de la démocratie. Actualité de la démocratie participative, Paris, Le Seuil.

Bonaccorsi J., Nonjon M., 2012. «La participation en kit»: l'horizon funèbre de l'idéal participatif, Quaderni, 79, 2944, https://doi.org/10.4000/quaderni.618.
Bryson J.M., Quick K.S., Schively Slotterback C., Crosby B. C., 2012. Designing Public Participation Processes, Public Administration Review, 73, 1, 23-34, https://doi.org/ 10.1111/j.1540-6210.2012.02678.x.

Callon M.,Law J., 1988. Laprotohistoired'unlaboratoire, in Callon M. (Ed.), La science et ses réseaux. Genèse et circulation des faits scientifiques, Paris, La Découverte, 66-116.

Carrel M., 2007. Pauvreté, citoyenneté et participation. Quatre positions dans le débat sur les modalités d'organisation de la 'participation des habitants' dans les quartiers d'habitat social, in Neveu C. (Ed.), Cultures et pratiques participatives, Perspectives comparatives, Paris, L'Harmattan, 95-112.

Chemery J-B., Gasc G., Arama Y., Dubois N., De la rocque J., Renoullin M., 2018. État des lieux des démarches participatives pour une gestion intégrée et durable de l'eau et des milieux aquatiques. Rapport final, Contrechamp, Cereg ingénierie, Agence française pour la biodiversité, http://www.gesteau.fr/document/etat-deslieux-des-demarches-participatives-pour-une-gestion-inte gree-et-durable-de-l'eau-e.

Cleaver F., Franks T., 2005. How institutions elude design: River basin management and sustainable livelihoods, $B C I D$ Research Paper, 12, https://bradscholars.brad.ac.uk/handle/ 10454/2964.

Contamin J.-G., Legris M., Spruyt É., 2017. La participation de la société civile à la recherche en matière d'environnement : les citoyens face au double cens caché participatif, Natures Sciences Sociétés, 25, 4, 381-392, https://doi.org/10.1051/ nss/2018006.

Crémin E., Linton J., Jacquin N., Perrin J.-A., 2018. Légitimité des savoirs citoyens dans la gestion participative des territoires de l'eau, Participations, 21, 117-141. https://doi. org/10.3917/parti.021.0117.

Daniell K.A., White I.M., Ferrand N., Ribarova I., Coad P., Rougier J.E., Hare M., Jones N.A., Popova A., Rollin D., Perez P., Burn S., 2010. Co-engineering participatory water management processes: Theory and insights from Australian and Bulgarian interventions, Ecology and Society, 15, 4, http://www.ecologyandsociety.org/vol15/iss4/art11/.

Debos F., Lacroix C., Cyrulnik N., Rasse P., Trousse B., 2017. Démarche de coconception citoyenne dans les processus d'innovation par les usages : le cas du projet Ecofamilies, Revue Information Organisation et Connaissance, 17, 1, https://doi.org/10.21494/ISTE.OP2019.0379.

Dionnet M., Imache A., Leteurtre E., Rougier J-E., Dolinska A., 2017. Guide de concertation territoriale et de facilitation, Montpellier, Lisode, http://www.lisode.com/wp-content/ uploads/2017/02/Lisode_Guide_concertation.pdf.

Durand S., Baret C., Krohmer C., 2018. La sociologie de la traduction comme grille de recherche-intervention: le cas d'un projet de prévention des risques psychosociaux dans un hôpital public, RIMHE: Revue Interdisciplinaire Management, Homme \& Entreprise, 30, 7, 3-28, https:// doi.org/10.3917/rimhe.030.0003.

Ferrand N., Noury B., 2017. Initial Guidelines on Stakeholders' Engagement and Year 1 Participatory Process in the PCS. SPARE WPT1 D.T. 2.1.2, Final report, Montpellier, 
https://spare.boku.ac.at/index.php/en/about-spare/initialguidelines-on-stakeholders-engagement-and-year-1-partici patory-process-in-the-pcs.

Ferrand N., Hassenforder E., Aquae-Gaudi W., 2021. L'approche COOPLAGE-Quand les acteurs modélisent ensemble leur situation, principes ou plans pour décider et changer durablement, en autonomie, Revue Science Eaux \& Territoires, 35, numéro spécial "Des démarches participatives pour penser ensemble la gestion de l'eau et des territoires", 14-23, http:/www.set-revue.fr/lapproche-coo plage-quand-les-acteurs-modelisent-ensemble-leur-situ ation-principes-ou-plans-pour.

Floc'Hlay B., Plottu E., 1998. Consultation ou co-décision? La question de la participation du citoyen à l'évaluation des projets publics, Metropolis, 76-79.

Girard S., 2012. La territorialisation de la politique de l'eau est-elle gage d'efficacité environnementale? Analyse diachronique de dispositifs de gestion des eaux dans la vallée de la Drôme (1970-2011). Thèse de doctorat en géographie, Lyon, ENS de Lyon-Université de Lyon.

Girard S., Rivière-Honegger A., 2014. Territorialisation de la politique de l'eau en France: enseignements à partir du cas de la vallée de la Drôme (1980-2013), Cahiers de l'Agriculture, 23, 129-137, https://doi.org/10.1684/ agr.2014.0689.

Hassenforder E., Ferrand N., Pittock J., Daniell K.A., Barreteau O., 2015. A participatory planning process as an arena for facilitating institutional bricolage-Example from the Rwenzori region, Uganda, Society \& Natural Resources: An International Journal, 28, 9, 995-1012, https://doi.org/10.1080/08941920.2015.1054977.

Hassenforder E., Pittock J., Barreteau O., Daniell K.A., Ferrand N., 2016. The MEPPP framework: A framework for monitoring and evaluating participatory planning processes, Environmental Management Journal, 57, 1, 79-96, https://doi.org/10.1007/s00267-015-0599-5.

Hassenforder E., Ferrand N., Girard S., 2021a. L'ingénierie de la participation: préparer et penser une démarche participative, Science Eaux \& Territoires, 35, numéro spécial “Des démarches participatives pour penser ensemble la gestion de l'eau et des territoires", 28-35, http://www.set-revue.fr/ lingenierie-de-la-participation-preparer-et-penser-unedemarche-participative.

Hassenforder E., Girard S., Petitjean C., Fermond C., 2021 b. La co-ingénierie de la participation: une expérience citoyenne sur la rivière Drôme. Annexes, https://hal. inrae.fr/hal-03178457.
Jakku E., Thorburn P.J., 2010. A conceptual framework for guiding the participatory development of agricultural decision support systems, Agricultural Systems, 103, 9, 675-682, https://doi.org/10.1016/j.agsy.2010.08.007.

Kensing F., Blomberg J., 1998. Participatory design: issues and concerns, Computer Supported Cooperative Work, 7, 167185, https://doi.org/10.1023/A:1008689307411.

Kujala S., 2003. User involvement: A review of the benefits and challenges, Behaviour \& Information Technology, 22, 1, 1-16, https://doi.org/10.1080/01449290301782.

Lyet P., 2011. Traduction, transaction sociale et tiers intermédiaire dans les processus de collaboration de chercheurs et de praticiens dans le cadre de recherches-actions, Pensée plurielle, 28, 49-67, https://doi.org/10.3917/pp.028.0049.

Mazeaud A., 2012. Administrer la participation: l'invention d'un métier entre valorisation du militantisme et professionnalisation de la démocratie locale, Quaderni, 79, 4558, https://doi.org/10.4000/quaderni.621.

OCDE, 2015. Stakeholder Engagement for inclusive water governance, OECD Studies on Water, Éditions OCDE, Paris, https://doi.org/10.1787/9789264231122-en.

Simon H.A., 1977. The new science of management decision [Revised edition], Englewood Cliffs, Prentice-Hall.

The World Bank, 1996. The World Bank Participation Source Book, Washington, D.C., https://documents1.worldbank. org/curated/en/289471468741587739/pdf/multi-page.pdf.

Tippett J., Searle B., Pahl-Wostl C., Rees Y., 2005. Social learning in public participation in river basin managementearly findings from HarmoniCOP European case studies, Environmental Science \& Policy, 8, 287-299, https://doi. org/10.1016/j.envsci.2005.03.003.

Valls-Donderis P., Ray D., Peace A., Stewart A., Lawrence A., Galiana F., 2013. Participatory development of decision support systems: which features of the process lead to improved uptake and better outcomes?, Scandinavian Journal of Forest Research, 29, Issue sup1 "Decision Support Systems for Sustainable Forest Management", 7183, https://doi.org/10.1080/02827581.2013.837950.

Vink P., Imada A.S., Zink K.J., 2008. Defining stakeholder involvement in participatory design processes, Applied Ergonomics, 39, 4, 519-526, https://doi.org/10.1016/j. apergo.2008.02.009.

Williams G., 2004. Evaluating participatory development: tyranny, power and (re)politicisation, Third World Quarterly, 25, 3, 557-578, https://doi.org/10.1080/ 0143659042000191438 .

Citation de l'article : Hassenforder É., Girard S., Ferrand N., Petitjean C., Fermond C. La co-ingénierie de la participation : une expérience citoyenne sur la rivière Drôme. Nat. Sci. Soc. 29, 2, 159-173. 